\title{
Patrones de distribución espacial del arbolado en un bosque de Pseudotsuga menziesiien Chihuahua, México
}

\section{Spatial distribution patterns of trees in a Pseudotsuga menziesii forest in Chihuahua, Mexico}

\author{
Samuel Alberto García-García!, Eduardo Alanís-Rodríguez*, Ernesto Alonso Rubio-Camacho², \\ Oscar Alberto Aguirre-Calderón', Eduardo Javier Treviño-Garza' y Gabriel Graciano-Ávila'
}

\begin{abstract}
1 Universidad Autónoma de Nuevo León. Facultad de Ciencias Forestales. Linares, Nuevo León, México.
\end{abstract}

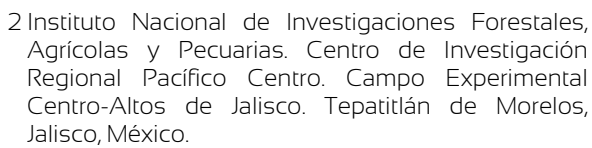

* Autor de correspondencia. eduardo.alanisrd@uanl.edu.mx 


\section{INTRODUCCIÓN}

Los bosques templados brindan una serie de servicios ecosistémicos como la regulación del clima, captura de carbono y contribuyen a la recarga de mantos acuíferos, entre otros (Solís, Blanco, Sáenz, Méndez y López, 2018). Sin embargo, algunos bosques presentan distintas alteraciosnes (Mápula, López, Vargas y Hernández., 2007; VelascoGarcía et al., 2007). Debido a ello, es necesario generar información sobre la estructura de estos bosques a escala de rodal, que brinde soporte para la toma de decisiones sobre su manejo, conservación y restauración.

La estructura del bosque puede ser caracterizada a nivel de diversidad de especies, dimensiones y a nivel espacial (Chávez-Flores, Corral-Rivas, Vega-Nieva, LópezSerrano y Rubio-Camacho, 2020; Gadow, Sánchez y Álvarez, 2007; Kulik, Molas y Rodríguez, 2019; Lin, Lam, Gadow y Kershaw, 2020; Mena-Mosquera, Andrade y Torres-Torres, 2019; Pommerening y Stoyan, 2020). Sin embargo, la investigación sobre estructura forestal en México ha sido enfocada a caracterizar la diversidad de especies y de dimensiones, siendo la distribución espacial una de las menos utilizadas, aunque brindan un mayor nivel de detalle. Estos estudios son importantes para determinar los cambios que originan los patrones y la variación espacial de la estructura del rodal, así como los efectos en la subsecuente sucesión de los ecosistemas forestales a escala de rodal (Yu, Wiegand y Yang 2009; Gu, Li, Qi y Wang, 2019).

Los análisis estructurales se han realizado a través de distintos índices y métodos que brindan información para la descripción de los ecosistemas; por ejemplo la estructura horizontal y vertical ha sido evaluada por medio del índice de valor de importancia (IVI), diagramas de perfil (Ogawa, Yoda, Ogino y Kira, 1965; Organización de las Naciones Unidas para la Educación, la Ciencia y la Cultura [Unesco], 1980), determinando pisos de altura (Lamprecht, 1990) o por medio del índice de $A$ (Pretzsch, 2009); mientras que las relaciones de vecindad se han descrito usando el método de muestreo de los cinco árboles, desarrollado para evaluar los atributos estructurales (dimensiones, especies y la regularidad de sus posiciones) de los árboles (Aguirre, Hui, Gadow y Jiménez, 2003; Gadow, Real y Álvarez, 2001).

En algunos estudios llevados a cabo tanto dentro de México como a escala internacional se ha evaluado la estructura y distribución espacial de comunidades forestales, tratando de responder a distintas preguntas de investigación. En bosques templados, por ejemplo, se han utilizado para caracterizar la estructura de rodales con algún grado de perturbación (Ávila-Flores, González-Tagle, Jiménez-Pérez, Aguirre-Calderón, Treviño-Garza, VargasLarreta y Alanís, 2014; Rubio-Camacho, González-Tagle, Himmelsbach, Ávila-Flores, Alanís-Rodríguez y JiménezPérez, 2017), también para comprender la distribución espacial de comunidades arbóreas como bosques de pino encino en Oaxaca y bosques subtropicales caducifolios en China (Castellanos-Bolaños, Treviño-Garza, AguirreCalderón, Jiménez-Pérez y Velázquez-Martínez, 2010; Du, Hu, Zeng, Wang, Peng, Zhang y Song, 2017), conocer relaciones interespecíficas entre especies (Wédjangnon, Kuiga, Houêtchégnon y Ouinsavi, 2020). Asimismo, se han utilizado para contrastar la estructura de bosques con y sin manejo forestal (García, Olivares y Ramos, 2013; García, Narváez, Olivas y Hernández, 2019; Hernández, Aguirre, Alanís, Jiménez, Treviño, González, Lujan, Olivas y Domínguez, 2013) y para diseñar patrones de plantación que aumenten la competencia asimétrica y la resistencia de los ecosistemas (Zhang, Hui, Hu, Zhao, Guan, Gadow y Zhang, 2019).

Sin embargo, existen escasos estudios que evalúen los patrones de distribución espacial en bosques de alto valor de conservación, que presentan comunidades vegetales con especies vulnerables. Un ejemplo de estos ecosistemas es el bosque de Pseudotsuga menæiesii, que tiene escasa distribución natural en México, encontrándose en la Sierra Madre Occidental y Oriental, así como en el centro - sur del país. Las poblaciones $P$. meñiesii se encuentran fragmentadas en rodales o manchones aislados, normalmente dominados por otras especies (Debreczy y Racz, 1995; DomínguezCalleros, Chávez-Flores, Rodríguez-Téllez, Corral-Rivas, Goche-Telles y Díaz-Vásquez, 2014; Rzedowski, 1978). 
Diversos factores amenazan los bosques de Pseudotsuga en México, ya que tiene problemas de regeneración y desarrollo (Torres, 2006; Mápula et al., 2007). Entre los factores más importantes se encuentran el cambio de uso de suelo, incendios, sobrepastoreo, tala clandestina y plagas (Mápula et al., 2007; Ventura, López, Vargas y Guerra, 2010; Velasco-García, López-Upton, Angeles-Pérez y Guerra-de la Cruz, 2007). Sin embargo, uno de los factores que más efectos podría tener es el cambio climático, ya que las poblaciones de Pseudotsuga meziesii emigraron hacia el sur durante las glaciaciones, por lo que son sensibles a los cambios de temperatura a escala global ocasionando, por un lado que las áreas con condiciones adecuadas para su desarrollo y establecimiento se reduzcan y contraigan hacia las partes más altas de los sistemas montañosos y hacia el hemisferio norte (Hermann, 1985; Mápula-Larreta, LópezUpton, Vargas-Hernández y Hernández-Livera, 2008). De ahí que el hábitat de Psendotsuga menzriesii esté compuesto de especies altamente especializadas, con alta biodiversidad y alto número de especies amenazadas (Schwendtner, Recalde, Alcalde, Gómez y Cárcamo, 2005).

\section{OBJETIVOS}

El objetivo de este estudio fue caracterizar los patrones de distribución espacial de un bosque maduro en el Estado de Chihuahua, México. Para ello, se plantearon las siguientes preguntas de investigación: 1) ¿La distribución del arbolado es aleatoria? 2) ¿La mezcla de especies es uniforme? 3) ¿Existe homogeneidad dimensional a nivel espacial? 4) ¿Cuáles son las principales diferencias y/o similitudes con otros estudios? Dar respuesta a estas preguntas proporcionará información básica para la toma de decisiones en cuanto al manejo, conservación y restauración de estos ecosistemas de alta importancia ecológica, económica y social.

\section{MATERIALES Y MÉTODOS}

\section{Área de estudio}

El estudio se llevó a cabo en el paraje "El Triste", ubicado en el ejido Chinatú, municipio de Guadalupe y Calvo, al suroeste del estado de Chihuahua. Geográficamente se encuentra entre las coordenadas $26^{\circ} 14^{\prime} 43^{\prime \prime}$ y $106^{\circ} 38^{\prime} 37^{\prime \prime}$ (Fig. 1). Dicho bosque tiene una superficie de 80.46 ha $^{-1} \mathrm{y}$ altitudes entre los $2530 \mathrm{~m}$ y $2830 \mathrm{~m}$ s.n.m. Pertenece a la Región hidrológica Río Fuerte, a su vez a la provincia fisiográfica Sierra Tarahumara y a la subprovincia Gran Meseta y Cañones Chihuahuenses. El tipo de suelo predominante en el área es litosol. El clima de la región está clasificado como templado subhúmedo (Cw), con temperatura media anual de $13.7{ }^{\circ} \mathrm{C}$ y una precipitación pluvial media anual de $1126.8 \mathrm{~mm}$ (Chávez, 2009). El bosque está conformado principalmente por $P$. menziesii, $P$. arizonica y Quercus tuberculata, con presencia de especies importantes para la conservación como Abies durangensis y Picea chibuahuana. Dicha área se encuentra catalogada como "Relicto de bosque viejo o sobremaduro" de acuerdo con Galván-Moreno (2016) y se encuentra excluida del aprovechamiento forestal en el programa de manejo.

\section{Toma de datos}

Se establecieron ocho sitios aleatorios circulares de $1000 \mathrm{~m}^{2}$ para el levantamiento de información. Dicho número de sitios se determinó de acuerdo con un pre-muestreo realizado y de acuerdo con una intensidad de muestreo de $1 \%$. Se consideraron individuos con diámetro normal (DN) $\left(d_{1.30}\right) \geq 7.5 \mathrm{~cm}$, además se registró la altura total $(\mathrm{h}, \mathrm{m})$, distancias de los árboles al centro del sitio, azimut y especie de cada individuo.

\section{Análisis de la información}

Se realizó el cálculo de la densidad (número de árboles por hectárea, ha $\left.{ }^{-1}\right)$, área basal $\left(\mathrm{m}^{2} \mathrm{ha}^{-1}\right)$, diámetro normal promedio $\left(d_{1.30}(\mathrm{~cm})\right)$ y altura total $(\mathrm{m})$ promedio para conocer de manera general las características dasométricas de cada uno de los géneros presentes en el bosque. La caracterización de los patrones de distribución espacial del bosque se obtuvo utilizando técnicas de análisis de patrón de puntos y relaciones de vecindad basados en el método de los cinco árboles (Albert, 1999; Hui y Hu, 2001) el cual toma un árbol de referencia (i) y sus cuatro vecinos más cercanos 


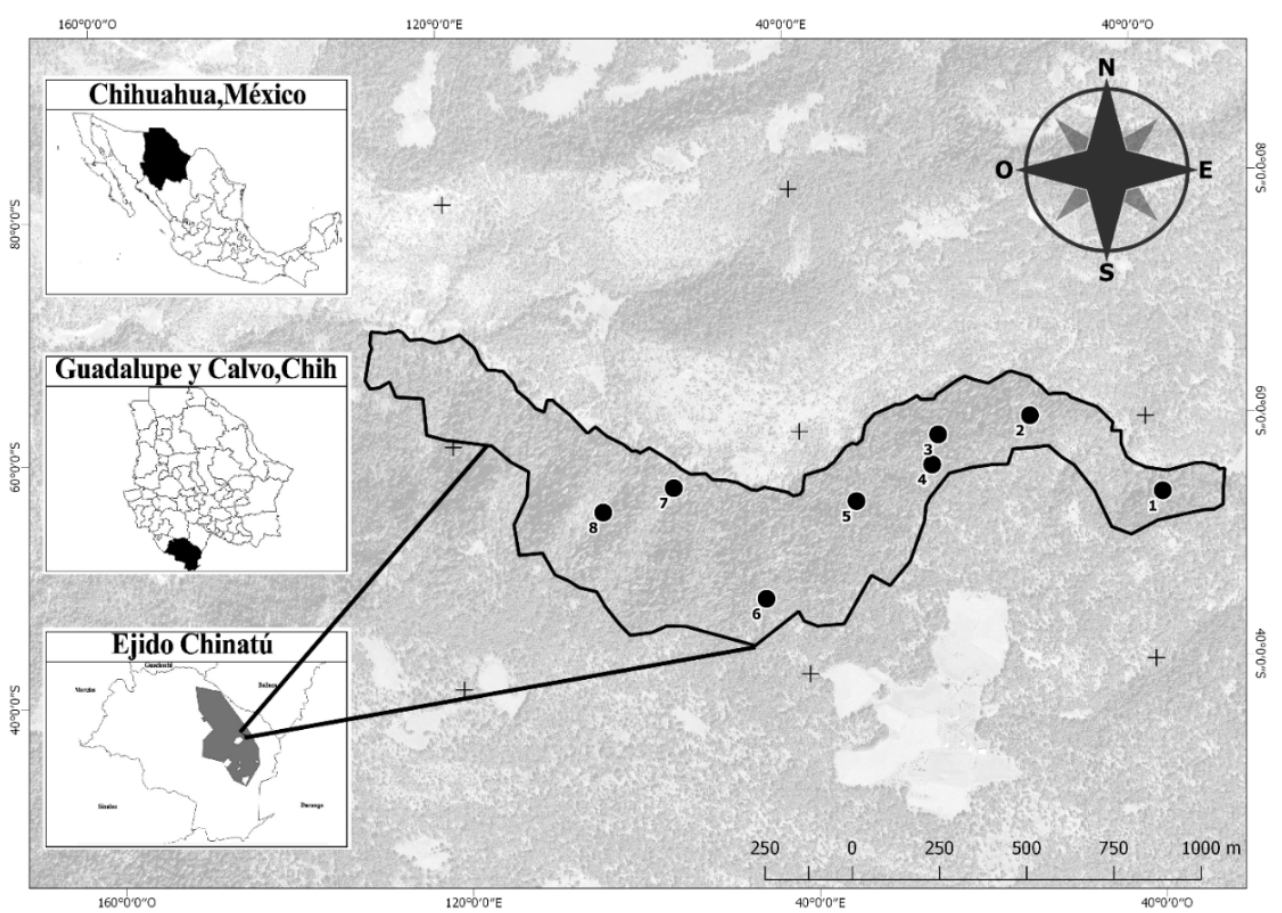

FIGURA 1. Localización del área de estudio y sitios de muestreo.

\section{Índice de uniformidad de ángulos $\left(W_{i}\right)$}

Se basa en la medición de los ángulos entre los vecinos a un árbol de referencia $i$ y su comparación con un ángulo estándar $\alpha$, de tal manera que al considerar cuatro vecinos al árbol de referencia $W_{i}$ puede tomar valores de 0 hasta 1 , donde un valor cercano a cero representa condiciones de regularidad, valores cercanos a 0.5 muestran tendencia a la aleatoriedad y los próximos a 1 presentan condiciones de agrupamiento (Gadow, Hui y Albert, 1998):

$$
W_{i}=\frac{1}{n} \sum_{j=1}^{n} V_{i j}
$$

\section{Donde:}

$W_{i}=\quad$ valor del índice para el $j$-ésimo árbol referencia

$\mathrm{n}=\quad$ es el número de árboles vecinos considerados

$V_{i j}=$ variable 1 cuando el $j$-ésimo ángulo $\alpha$ entre dos árboles vecinos próximos es menor o igual al ángulo estándar $\alpha$, en caso contrario toma un valor de 0 .

\section{Grado de mezcla en la distribución espacial de especies $\left(M_{i}\right)$}

Se calculó con base en el índice de mezcla de especies $\left(M_{i}\right)$ el cual es una medida de la diversidad en la distribución espacial de especies mostrando el grado de mezcla entre estas, Füldner (1995) define este índice como la proporción de $n$ vecinos más cercanos que no pertenecen a la misma especie que el árbol de referencia, en este caso se toman los cuatro vecinos más cercanos.

$$
M_{i}=\frac{1}{n} \sum_{j=1}^{n} v_{j}
$$

Donde $0 \leq M i \leq 1, v_{j}$ es igual a 0 cuando el árbol $j$ es de la misma especie que el árbol de referencia $i$ y será 1 en caso contrario (Gadow et al., 2007).

\section{Dominancia dimensional $\left(U_{i}\right)$}

Se define como la dominancia del árbol referencia $i$ con respecto a sus vecinos más cercanos, es decir, la proporción 
de árboles $n$ más pequeños (en diámetro o altura) que el árbol de referencia.

$$
U_{i}=\frac{1}{n} \sum_{j=1}^{n} v_{j}
$$

Donde $0 \leq U_{i} \leq 1$ y $v_{j}$ será igual a 1 si el árbol $j$ es menor al árbol referencia $i$, y 0 en caso contrario. Con cuatro vecinos el índice de dominancia $U_{i}$ puede tomar cinco valores y es muy útil cuando se desea ver la dominancia relativa de una especie en particular (Aguirre, Hui, von Gadow y Jiménez, 2003; Gadow et al., 2007).

\section{Diferenciación dimensional $\left(T_{i}\right)$}

Se puede aplicar a cualquier variable representativa de las dimensiones del arbolado, en este caso para diámetros y alturas. El grado de diferenciación dimensional establece una manera de representación de la proximidad espacial que existe entre árboles de diferentes dimensiones en el bosque. Toma en cuenta los $n$ árboles más cercanos al árbol de referencia $i$ y se define de la siguiente manera:

$$
T_{i}=1 \frac{1}{n} \sum_{j=1}^{n} \frac{\min \left(\operatorname{Dim}_{i}, \operatorname{Dim}_{j}\right)}{\operatorname{Max}\left(\operatorname{Dim}_{i}, \operatorname{Dim}_{j}\right)}
$$

\section{Donde:}

$T_{i}=\quad$ grado de diferenciación en diámetros $\mathrm{o} / \mathrm{y}$ alturas

$j=1 . . n$ árboles vecinos

$i=\quad$ árbol de referencia

$\operatorname{Dimi}_{i}=$ diámetro o altura del árbol i

$D i m_{j}=$ diámetro o altura del árbol $j$

El valor del $T_{i}$ se incrementa al aumentar la diferencia media del tamaño de los árboles vecinos $\left(0 \leq T_{i} \leq 1\right)$. Entre más bajo sea el valor de $T_{i}$ las diferencias son menores, un valor de 0 indica que todos los árboles son del mismo tamaño (Gadow et al., 2007). Siguiendo el método de Aguirre, Kramer y Jiménez (1998), el grado de diferenciación en diámetros y alturas se clasificó en cinco categorías, 0-0.2 = débil; 0.2-0.4 = moderada; 0.4-0.6 clara; 0.6-0.8 fuerte; 0.81.0 muy fuerte.

El cálculo de los índices se ejecuta dentro de una ventana de observación $(W)$, que esta vez son los sitios circulares de muestreo. En dichos sitios se realiza cada uno de los análisis de la información con las fórmulas previamente descritas. Considerándose el efecto de borde de la ventana (Pommerening y Stoyan, 2006), este efecto desempeña un papel importante para la correcta caracterización de la estructura espacial.

Para una correcta interpretación de las características estructurales en el espacio se debe tomar en cuenta el efecto de borde, ya que hay árboles dentro de los sitios de muestreo que se encuentran cerca del borde $W$ y normalmente tienen a sus vecinos más cercanos del otro lado. Lo anterior implica errores en la estimación de los valores de vecindad de los árboles que se encuentran dentro. Con la finalidad de evitar errores se utilizó el estimador NN1 el cual consiste en omitir como árbol de referencia (i) a aquellos cuya distancia a su $j$-ésimo vecino más cercano sea menor a la distancia entre este y el borde de $W$, pese a esto pueden formar parte de otros grupos estructurales como vecino más cercano (Pommerening y Stoyan, 2006).

Con el objetivo de comparar estadísticamente los índices antes mencionados con bosques perturbados o bajo manejo, se amplió la base de datos a 1000 submuestras utilizando la paquetería "WRS2" en el programa " $R$ " (R Core Team, 2019), mediante la cual se usaron simulaciones "Bootstrap" para calcular la diferencia entre medias con un IC de 95\% (Mair, Wilcox y Schoenbrodt, 2015; Wilcox, 2011).

\section{Resultados}

Los resultados de distribución espacial se muestran para el bosque en general y por géneros (Psendotsuga, Quercus y Pinus), por ser los más abundantes, de mayores dimensiones y más investigados en este aspecto. La densidad total fue de $254 \mathrm{ha}^{-1}$ con un área basal de $10.14 \mathrm{~m}^{2} \mathrm{ha}^{-1}$ siendo Pseudotsuga el género más importante (Tabla 1). 
TABLA 1. Características dasométricas del área de estudio.

\begin{tabular}{lllll}
\hline Género & $\begin{array}{l}\text { Densidad } \\
\left(\mathrm{ha}^{-1}\right)\end{array}$ & $G\left(\mathrm{~m}^{2} \mathrm{ha}^{-1}\right)$ & $d_{1.30}(\mathrm{~cm})$ & $h(\mathrm{~m})$ \\
\hline Pseudotsuga & 105 & 3.4780 & 21.24 & 11.04 \\
Quercus & 28 & 1.7196 & 21.45 & 10.94 \\
Pinus & 53 & 1.6963 & 21.30 & 10.99 \\
Arbutus & 34 & 2.2618 & 21.29 & 10.96 \\
Juniperus & 16 & 0.5176 & 21.27 & 10.82 \\
Abies & 13 & 0.2504 & 21.71 & 10.92 \\
Picea & 6 & 0.2194 & 21.87 & 10.72 \\
\hline Total & $\mathbf{2 5 4}$ & 10.143 & & \\
\hline
\end{tabular}

$\mathrm{G}$ = área basal, d.30 = diámetro normal promedio, $\mathrm{h}$ = altura promedio.

\section{Distribución espacial del arbolado}

En la figura 2 se muestra la distribución espacial del arbolado en cada uno de los sitios después de usar el estimador NN1, es decir, únicamente se muestran los árboles que pueden ser el árbol $i$ del grupo estructural.
Como resultado, en el análisis de los índices de vecindad se utilizaron un total de 203 grupos estructurales de los cinco árboles para el bosque en general, siendo los sitios 2 y 6 donde se conformó una mayor cantidad de grupos, 41 y 54 respectivamente; Pseudotsuga generó 84 grupos estructurales, Quercus 22 у Pinus 42.
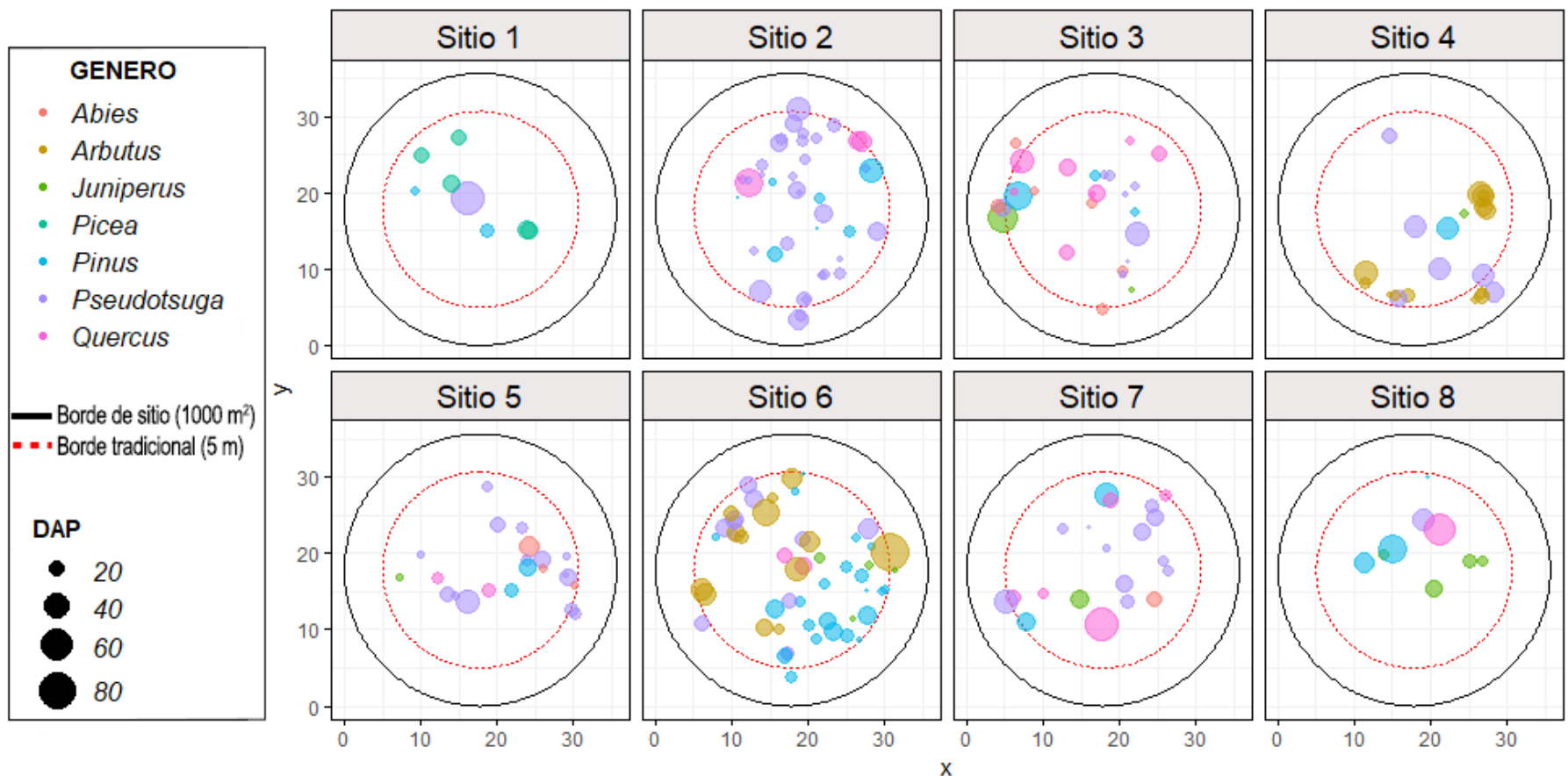

FIGURA 2. Distribución espacial del arbolado en cada uno de los sitios por género. 


\section{Uniformidad de ángulos $\left(W_{i}\right)$}

En el análisis general, los resultados muestran una media $(\overline{\mathbf{X}})$, generada con la simulación Bootstrap, de 0.49 con un intervalo de confianza [0.45-0.53], evidenciando así una distribución aleatoria. En la figura 3 se puede observar que la mayoría de los grupos estructurales se ubican en la categoría 0.5, indicando que dos de los cuatro árboles vecinos tienen un ángulo menor a $90^{\circ}$. De igual manera el análisis por género presentó una distribución aleatoria, Psendotsuga $(\overline{\mathbf{X}}=0.44$ [0.37-0.50]); mientras que Quercus y Pinus presentaron $(\overline{\mathbf{X}}=0.47[0.39-0.53])$ y $(\overline{\mathbf{X}}=0.49[0.40$ 0.57]) respectivamente, con ligera tendencia a una distribución regular.

\section{Índice de mezcla de especies $\left(M_{i}\right)$}

Se obtuvo $(\overline{\mathbf{X}}=0.83$ [0.76-0.89]) para el bosque en general, teniendo el mayor porcentaje en las categorías 0.75 y 1 (Fig. 4), lo que indica que la mayoría de los árboles de referencia (i) están rodeados por individuos de especies diferentes a él. El análisis por género mostró que Pseudotsuga $(\overline{\mathbf{X}}=0.65[0.48$ 0.83]) suele estar o no mezclada con individuos de cualquier especie y la mayoría de sus individuos se encuen-tran en la categoría 0.5, Quercus ( $\overline{\mathbf{X}}=0.90$ [0.83-0.97]) normalmente aparece mezclado con diferentes especies ya que la mayoría de sus individuos se encuentran en las categorías 0.75 y 1 , mientras que Pinus $(\overline{\mathbf{X}}=0.90$ [0.80-0.98]) tiene una ligera tendencia a estar mezclado con especies diferentes, con la mayor frecuencia en las categorías 0.75 y 1.

\section{Índice de dominancia dimensional diamétrica $\left(U D_{i}\right)$}

Las distribuciones de los valores de dominancia relativa no muestran ninguna tendencia en el análisis general del bosque (Fig. 5) ( $\overline{\mathbf{X}}=0.53$ [0.45-0.61]); mientras que, al analizarse por género, Pseudotsuga $(\overline{\mathbf{X}}=0.59$ [0.49-0.73]) tiene la mayor proporción individuos en la categoría de 0.75 , lo que indica que es una especie que domina sobre las que la rodean. Quercus $(\overline{\mathbf{X}}=0.56$ [0.40-0.75]), de igual manera tiene la mayor frecuencia en la categoría 1, sin embargo, las categorías menores 0 y 0.25 cuentan con alrededor de $45 \%$ de los individuos, mostrando así que este género puede tener árboles tanto dominantes como dominados, mientras que Pinus $(\overline{\mathbf{x}}=0.55$ [0.37-0.72] ) al contrario tiene la mayoría de las frecuencias en la categoría 0 , más de $25 \%$, sin embargo, las categorías 0.75 y 1 suman más de $40 \%$, indicando que normalmente se encuentra rodeado por árboles más grandes, tanto en diámetro como en altura, pero puede llegar a dominar el algunas ocasiones.

\section{Índice de dominancia dimensional de altura $\left(U H_{i}\right)$}

De manera general $(\overline{\mathbf{x}}=0.51$ [0.43-0.59]) la distribución de dominancia en altura muestra una ligera tendencia a que existen especies dominantes en el área de estudio (Fig. 6), eso se corrobora en el análisis por género ya que Pseudotsuga $(\overline{\mathbf{X}}=0.64[0.53-0.76])$ tiene su mayor frecuencia en las categorías 0.75 y 1 , indicando que es una especie dominante en altura. Quercus $(\overline{\mathbf{X}}=0.45$ [0.33-0.58]), tienen las mayores frecuencias en las categorías menores por lo que se encuentran rodeados por árboles más grandes, Pinus $(\overline{\mathbf{X}}=$ 0.62 [0.48-0.75]) por su parte tuvo sus mayores frecuencias en la categoría de 0.50 y 0.75 , indicando que es una especie codominante.

\section{Índice de diferenciación dimensional diamétrica $\left(T D_{i}\right)$}

El bosque muestra una diferenciación diamétrica moderada, teniendo alrededor de $60 \%$ de frecuencia en esta categoría (Fig. 7), seguida de la categoría clara. El análisis por género mostró la misma tendencia (Moderada). Por otra parte, los valores medios obtenidos mediante la prueba Bootstrap quedaron dentro del intervalo de confianza para todos los grupos.

\section{Índice de diferenciación dimensional de altura $\left(T H_{i}\right)$}

$\mathrm{Al}$ igual que en la diferenciación diamétrica la distribución de alturas se muestra similar presentando la mayoría de sus frecuencias en la categoría moderada, seguida de la clara (Fig. 8). El análisis por género mostró la misma tendencia y los valores medios obtenidos mediante la prueba Bootstrap quedaron dentro del intervalo de confianza para todos los grupos. 

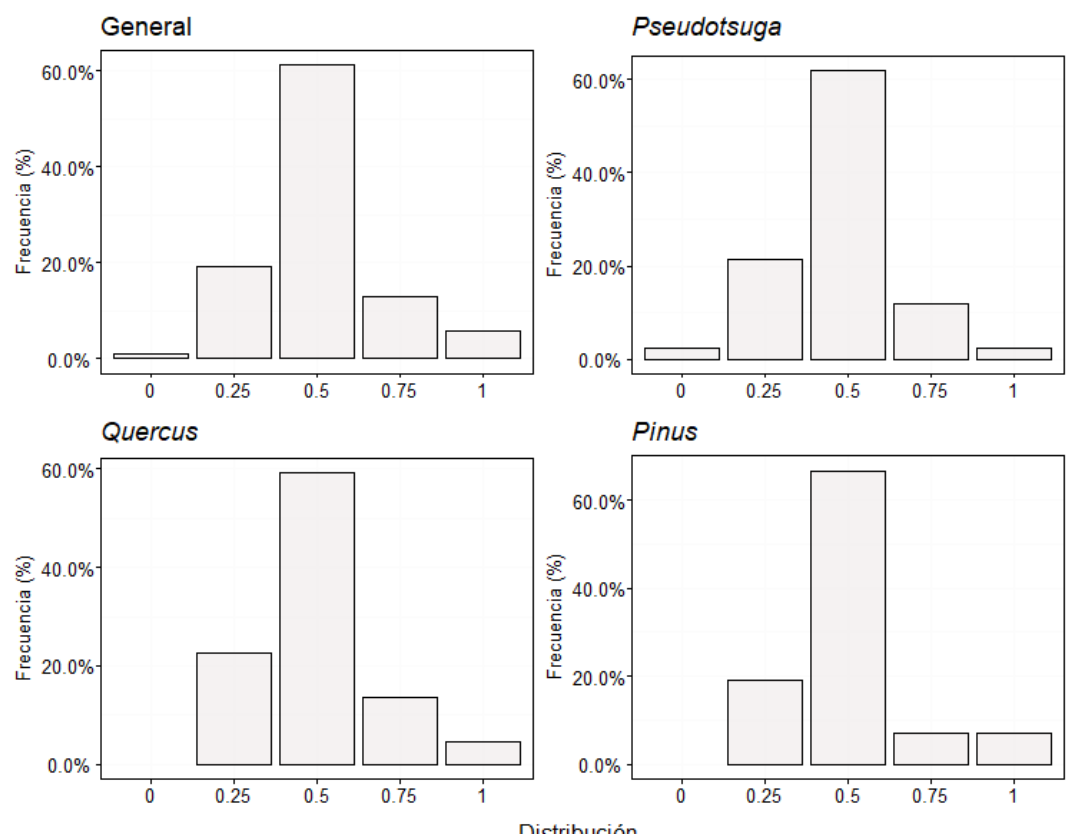

$(0=$ Regular; 0.5 = Aleatoria; 1 = Agregada $)$

FIGURA 3. Distribución del índice de uniformidad de ángulos $\left(W_{i}\right)$ general y por género: Pseudotsuga, Quercus y Pinus. El grupo general incluye a todos los géneros.
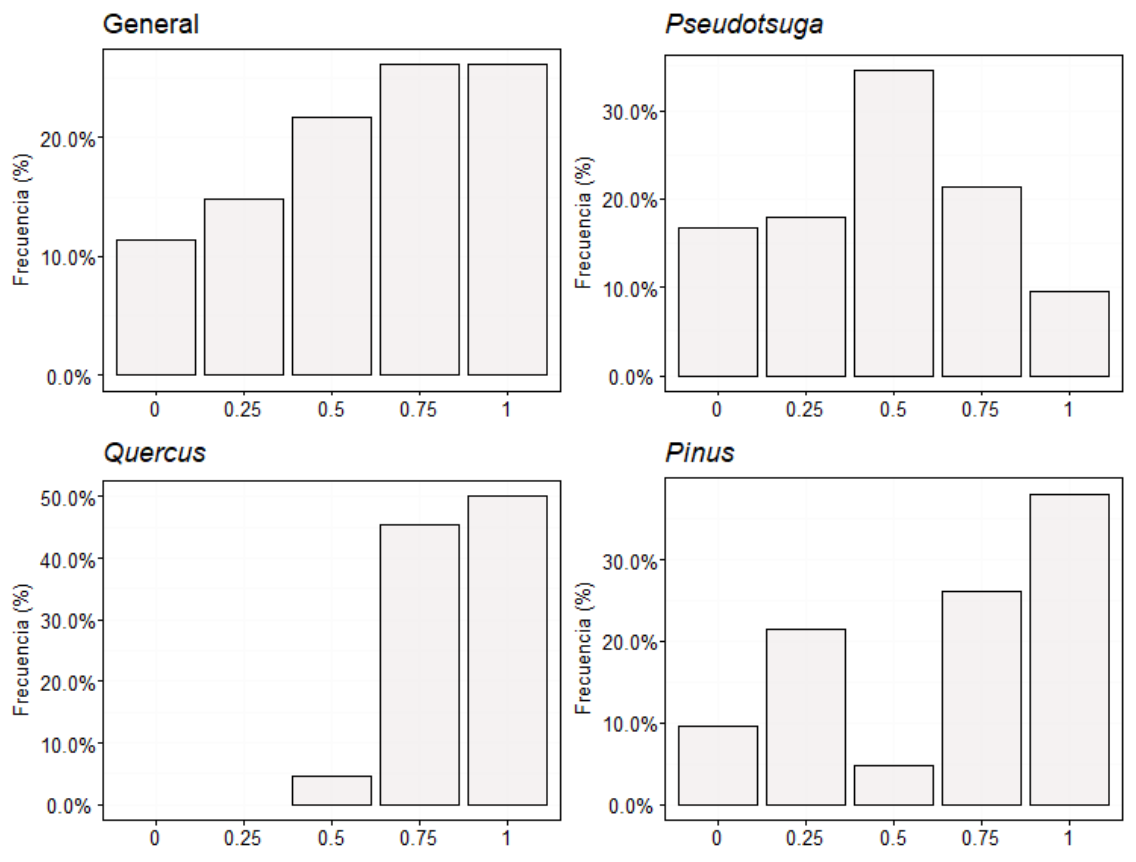

$(0=$ Nulo; $0.5=$ Medio; 1 = Alto $)$

FIguRA 4. Distribución del índice de mezcla de especies $\left(M_{i}\right)$ general y por género. 

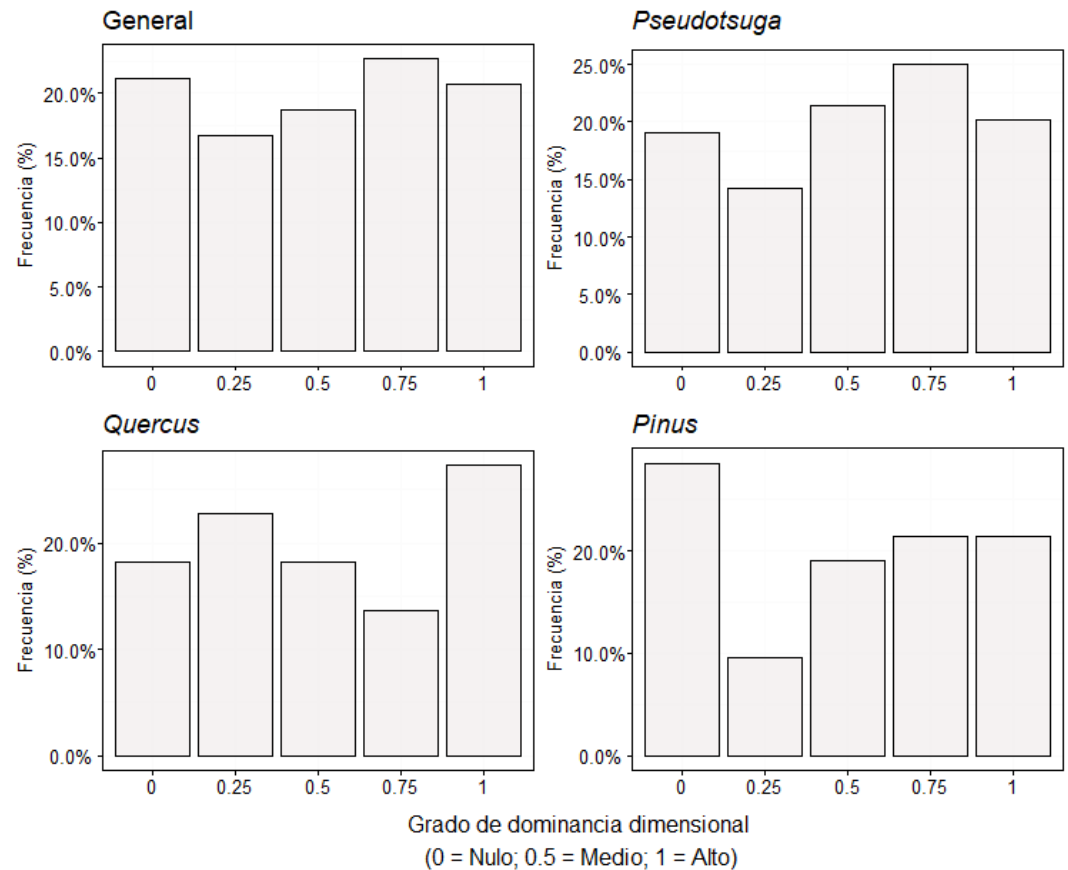

FIGURA 5. Distribución del índice de dominancia dimensional diamétrica $\left(U D_{i}\right)$ general y por género.
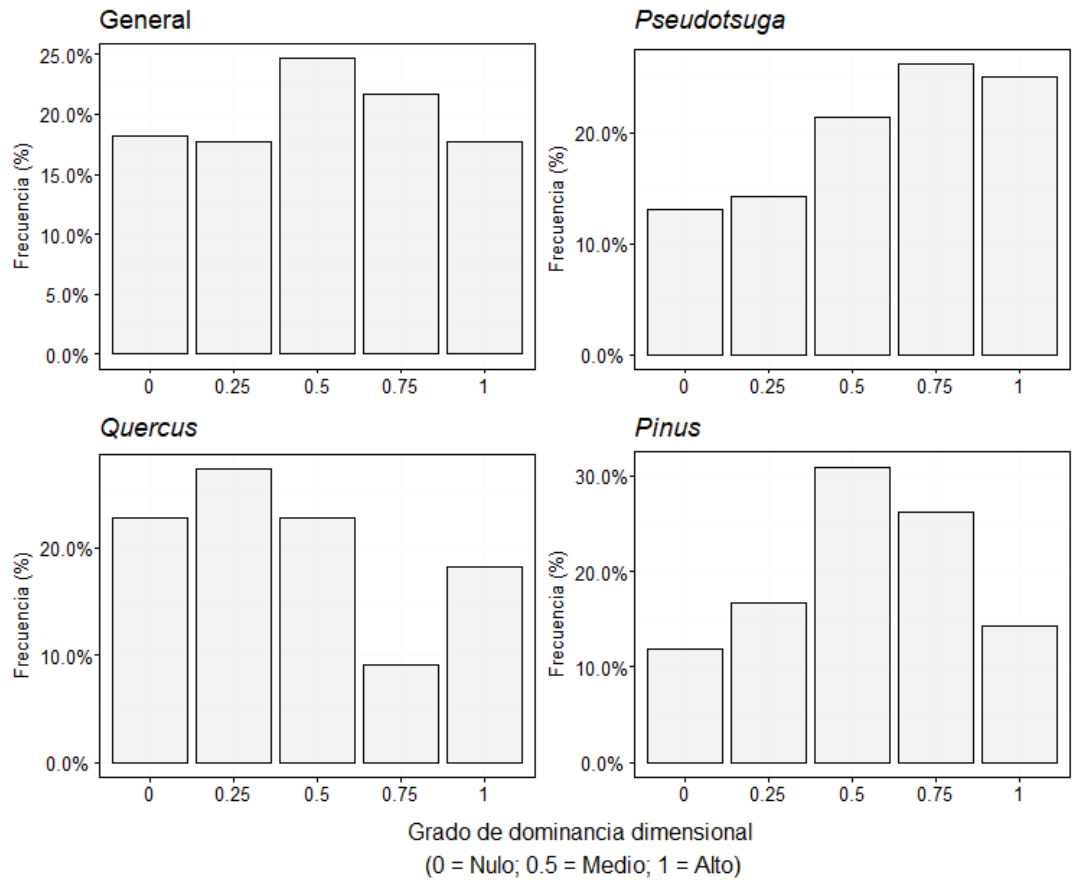

FIGURA 6. Distribución del índice de dominancia dimensional de altura $\left(\mathrm{UH}_{i}\right)$ general y por género. 

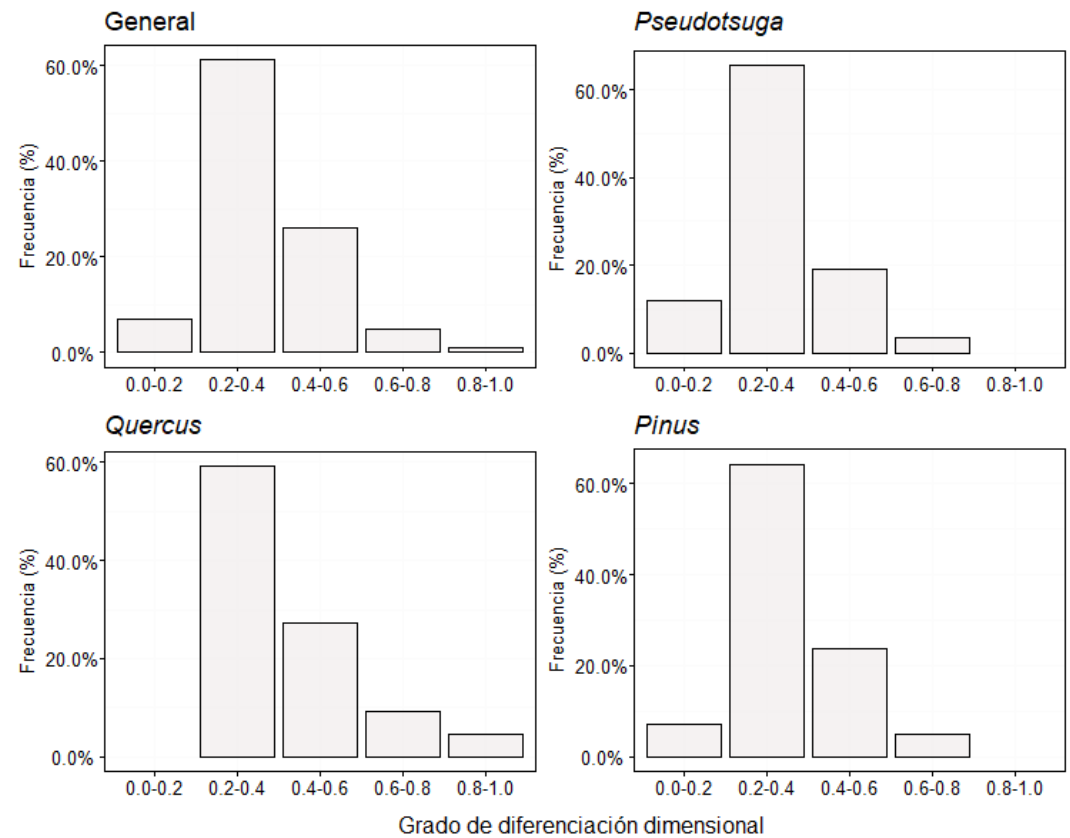

(0.0-0.2 = Débil; $0.2-0.4=$ Moderada; $0.4-0.6$ = Clara 0.6-0.8 = Fuerte; $0.8-1.0=$ Muy fuerte $)$

FIGURA 7. Distribución del índice de diferenciación dimensional diamétrica $\left(T D_{i}\right)$ general y por género.
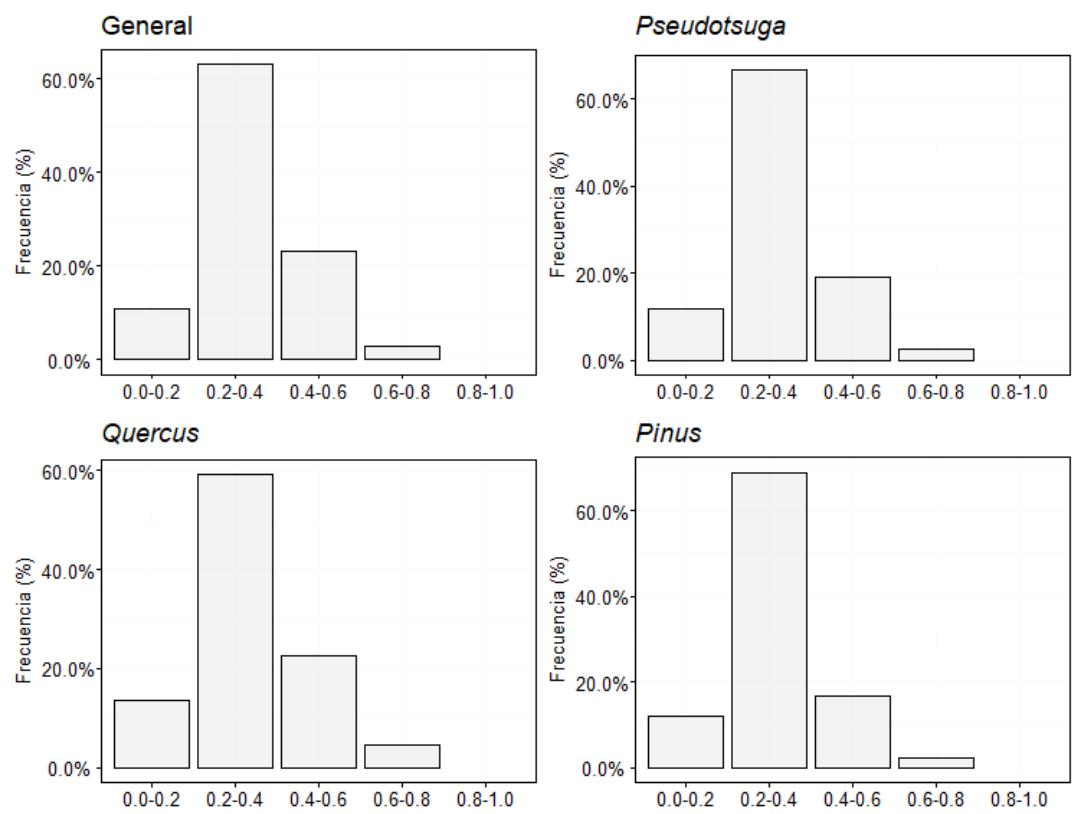

Grado de diferenciación dimensional

(0.0-0.2 = Débil; $0.2-0.4=$ Moderada; $0.4-0.6=$ Clara 0.6-0.8 = Fuerte; $0.8-1.0=$ Muy fuerte $)$

FIGURA 8. Distribución del índice de diferenciación dimensional de altura $\left(\mathrm{TH}_{i}\right)$ general y por género. 


\section{DISCUSIÓN}

Respondiendo a las preguntas de investigación planteadas la distribución y mezcla del arbolado fue aleatoria, asimismo el bosque presentó una tendencia a la homogeneidad ya que la diferenciación dimensional fue de moderada a clara. Una de las principales similitudes con otros estudios se reflejó en el índice de Uniformidad $\left(W_{i}\right)$ aplicado también en bosques naturales, en bosques bajo manejo y en bosques con antecedentes de incendios (Graciano-Ávila, AlanísRodríguez, Rubio-Camacho, Valdecantos-Dema, AguirreCalderón, González-Tagle y Mora-Olivo, 2020; RubioCamacho et al., 2017; Zhang et al., 2019). Mientras que una de las diferencias se presentó en el índice de mezcla de especies $\left(M_{i}\right)$ el cual fue distinto a lo encontrado en bosques de pino - encino y a otras poblaciones de Pseudotsuga (Domínguez-Calleros et al., 2014; Rubio-Camacho et al., 2017).

La estructura espacial del bosque es el resultado de dinámicas pasadas e interacciones complejas entre muchos procesos (Marie-Agnès y Audrey, 2003). Esta dinámica incluye la heterogeneidad ambiental y la competencia entre individuos y poblaciones (Duncan, 1991; Valverde y Silvertown, 1997). Aguirre et al. (2003) mencionan que este tipo de descripciones son más completas que los enfoques tradicionales.

\section{Distribución espacial del arbolado}

Los grupos estructurales generados $\left(254 \mathrm{ha}^{-1}\right)$ para este tipo de bosque fueron superiores a los creados por GracianoÁvila, Alanís-Rodríguez, Aguirre-Calderón, GonzálezTagle, Treviño-Garza y Mora-Olivo, (2019) para una parcela con tratamiento de regeneración $\left(96 \mathrm{ha}^{-1}\right)$ y otra con tratamiento de selección $\left(134 \mathrm{ha}^{-1}\right)$ para un bosque de pino - encino, mientras que Rubio-Camacho et al. (2017) generaron $243 \mathrm{ha}^{-1}$ grupos para una zona con evidencias de incendio y $281 \mathrm{ha}^{-1}$ para una sin evidencias de incendios en un bosque pino-encino. La mayoría de las especies arbóreas en los bosques de todo el mundo se agregan espacialmente a diferentes escalas y los patrones espaciales de los árboles cambian con la etapa de desarrollo del bosque, particularmente en los bosques naturales no muy perturbados por acción antropogénica (Condit, Ashton, Baker, Bunyavejchewin, Gunatilleke, Gunatilleke y Yamakura, 2000; Getzin et al., 2008; Yang et al., 2018).

\section{Índice de uniformidad de ángulos $\left(W_{i}\right)$}

Dicho índice evidenció una distribución aleatoria del bosque. Los resultados fueron similares a los calculados por Zhang et al. (2019), quienes evaluaron bosques naturales de Picea schrenkiana, Pinus koraiensis y un bosque mixto de pino - roble en diferentes regiones de China. Rubio-Camacho et al. (2017) registraron también esta distribución en bosques regenerados después de los incendios forestales. Mientras que Graciano-Ávila et al., (2020) lo documentaron para bosques bajo aprovechamiento forestal.

Los resultados de Quercus y Pinus no presentaron diferencias significativas $(\mathrm{p} \geq 0.05)$ contra estudios en bosques similares (Rubio-Camacho et al., 2017), ya que sus valores están entre 0.47 y 0.50 quedando dentro del intervalo de confianza (IC) de 95\%, Pseudotsuga por su parte no presentó diferencias con los valores registrados (S1: 0,509 y S2: 0,532) por Domínguez-Calleros et al. (2014).

\section{Índice de mezcla de especies $\left(M_{i}\right)$}

De manera general indica que los árboles referencia ( () están rodeados por individuos de especies diferentes a él. El análisis por género revela que Pseudotsuga no muestra preferencia por estar o no mezclado, contrario a lo publicado por Domínguez-Calleros et al. (2014), quienes observaron una mezcla alta para dos poblaciones de Pseudotsuga menziesii en la reserva de la biosfera "La Michilía" en los estados de Durango y Zacatecas. Quercus y Pinus muestran un mayor grado de mezcla en este estudio que en bosques de pino - encino (Rubio-Camacho et al., 2017).

Los resultados generales para mezcla de especies presentaron diferencias significativas comparado con lo mencionado por Rubio-Camacho et al. (2017), ya que sus valores medios fueron P1: 0.57 y P2: 0.58. Lo anterior se corrobora en el análisis por género ya que los mismos autores mencionan valores de P1: 0.49 y P2: 0.56 para Pinus y Quercus respectivamente quedando fuera del IC calculado 
(Pinus: 0.83-0.97, Quercus: 0.80-0.98). Pseudotsuga de igual manera presentó diferencias estadísticamente significativas ( $\mathrm{p} \geq 0.05)$ con lo observado (S1: 0,732 y S2: 0,665$)$ por Domínguez-Calleros et al. (2014).

\section{Índice de dominancia dimensional diamétrica $\left(U D_{i}\right)$}

No mostró ninguna tendencia para el bosque en general. Estos resultados al igual que los de Graciano-Ávila et al., (2019) y Rubio-Camacho et al. (2017) determinan que el bosque es heterogéneo en este aspecto, sin embargo, el análisis por género muestra que Pseudotsuga es dominante sobre las demás especies ya que este género es de los más abundantes y de mayores dimensiones (Diámetro normal, cobertura de copa) en el área de estudio (García et al., 2019). Quercus mostró una alta dominancia sobre las demás especies, aunque tiene un gran porcentaje en las categorías menores, Pinus por su parte se comportó como especie dominada contrario a lo observado por Rubio-Camacho et al. (2017).

\section{Índice de dominancia dimensional de altura $\left(U H_{i}\right)$}

Manifestó que Pseudotsuga es el género dominante en el área de estudio lo cual se entiende, ya que, de acuerdo con García et al., (2019) sus individuos son abundantes y de alturas máximas de hasta $26.30 \mathrm{~m}$. Al igual que en el estudio de Rubio-Camacho et al. (2017) Quercus es dominado por los demás géneros, mientras que Pinus es codominante según la clasificación de Gadow et al. (2007). Los resultados generales mostraron diferencias significativas $(p \leq 0.05)$ contra la parcela 1 estudiada por Rubio-Camacho et al. (2017), quienes obtuvieron una media de 0.35 , mientras que para la parcela 2 no existieron diferencias donde el valor fue de 0.45 encontrándose dentro del IC (0.43-0.59).

\section{Índice de diferenciación dimensional diamétrica y en altura $\left(T D_{i}\right.$ y $\left.T H_{i}\right)$}

El grado de diferenciación dimensional tanto en diámetros como en alturas fue moderado, similar a lo encontrado por Rubio-Camacho et al. (2017) y Graciano-Ávila et al. (2019) en bosques de pino - encino. Esto indica que existe escasa variabilidad en las dimensiones del arbolado y que la estructura, tanto horizontal como vertical es poco heterogénea.

\section{CONCLUSIONES}

Los resultados de esta investigación indican que la uniformidad $\left(W_{i}\right)$, en este caso aleatoria, no es exclusiva de bosques sin disturbio o sin manejo, además se observa que la mezcla de especies $\left(M_{i}\right)$ no necesariamente es mayor en bosques maduros y sin intervención como en este caso. Por su parte la dominancia diamétrica $\left(U D_{i}\right)$ muestra que géneros como Pinus pueden llegar a ser dominados por otros, así como la dominancia en altura $\left(\mathrm{UH}_{i}\right)$ corrobora que Quercus es un género dominado. Por su parte la diferenciación dimensional, en otros tipos de bosques es homogénea. Lo obtenido en este estudio deja en claro que existe necesidad de generar información de la estructura y dinámica espacial, de este tipo de ecosistemas ya que es escasa. La aplicación de los índices aquí empleados contribuye de manera importante al entendimiento de los procesos que se llevan a cabo en un bosque para llegar a generar una estructura. Lo anterior da bases para el manejo y conservación de comunidades vulnerables como la aquí estudiada, teniendo conocimiento de manera más individualizada de la dinámica a nivel especie o género.

\section{RECONOCIMIENTOS}

Al Consejo Nacional de Ciencia y Tecnología por la beca otorgada al primer autor. Al M.C. Raúl Narváez Flores por su apoyo en la identificación de especies.

\section{REFERENCIAS}

Aguirre, O., Hui, G., Gadow, K. von., \& Jiménez, J. (2003). An analysis of spatial forest structure using neighbourhood-based variables. Forest Ecology and Management 183, 137-145. doi.org:10.1016/S0378-1127(03)00102-6

Aguirre, O., Kramer, H., \& Jiménez, J. (1998). Strukturuntersuchungen ineinem Kiefern-Durchforstungsversuch Nordmexikos. Allgemeine Forst und Jagdzeitung, 169, 213-220.

Albert, M. (1999). Analyse der eingriffsbedingten Strukturveränderung und Durchforstungsmodellierung in Mischbeständen. Tesis doctoral. University Göttingen. Göttingen, Alemania. 
Ávila-Flores, D. Y., González-Tagle, M. A., Jiménez-Pérez, J., AguirreCalderón, O. A., Treviño-Garza, E., Vargas-Larreta, B., \& Alanís Rodríguez, E. (2014). Effect of the severity of fire in the structure characteristics of conifer forest stands. Revista Chapingo SerieCciencias Forestales y del Ambiente, 20(1), 34-45. doi: 10.5154/r.rchscfa.2013.01.005

Castellanos-Bolaños. J. F., Treviño-Garza. E. J., Aguirre-Calderón. O. A., Jiménez-Pérez. J., \& Velázquez-Martínez. A. (2010). Diversidad arbórea y estructura espacial de bosques de pinoencino en Ixtlán de Juárez, Oaxaca. Revista Mexicana de Ciencias Forestales, 1, 39-52. doi: 10.29298/rmcf.v1i2.636

Chávez, N. (2009). Estudio regional forestal de la Unidad de Manejo Forestal No.0808 "Guadalupe Y Calvo, Chibuabua". Asociación Regional de Silvicultores de Guadalupe y Calvo A.C. Recuperado de http://www.conafor.gob.mx:8080/documentos/docs/9/1147E RF_UMAFOR0808.pdf

Chávez-Flores, G., Corral-Rivas, J., Vega-Nieva, J., López-Serrano, P., \& Rubio-Camacho, E. (2020). Estructura espacial de los bosques mixtos e irregulares en el estado de Durango. Revista Mexicana de Ciencias Forestales, 11(59). doi: 10.29298/rmcf.v11i59.614

Condit, R., Ashton, P. S., Baker, P., Bunyavejchewin, S., Gunatilleke, S., Gunatilleke, N., ... \& Yamakura, T. (2000). Spatial patterns in the distribution of tropical tree species. Science, 288(5470), 1414-1418. doi: 10.1126 / science.288.5470.1414

Debreczy, Z., \& Rácz, I. (1995). New species and varieties of conifers from Mexico. Phytologia, 78(4), 217-243. doi: 10.5962/bhl.part.11916

Domínguez-Calleros, P. A., Chávez-Flores, G. A., Rodríguez-Téllez, E., Corral-Rivas, J. J., Goche-Telles, J. R., \& Díaz-Vásquez, M. A. (2014). Caracterización silvícola de Pseudotsuga menziesii en la reserva de la biosfera" La Michilía". Madera y Bosques, 20(2), 23-31. doi: $10.21829 /$ myb.2014.202161

Du, H., Hu, F., Zeng, F., Wang, K., Peng, W., Zhang, H., ... \& Song, T. (2017). Spatial distribution of tree species in evergreen-deciduous broadleaf karst forests in southwest China. Scientific Reports, 7(1), 1-9. doi: 10.1038/s41598-017-15789-5

Duncan, R. P. (1991). Competition and the coexistence of species in a mixed podocarp stand. The Journal of Ecology, 1073-1084. doi: 10.2307 / 2261099

Chávez-Flores, G. A., Corral-Rivas, J. J., Vega-Nieva, J. D., LópezSerrano, P. M., \& Rubio-Camacho, E. A. (2020). Estructura espacial de los bosques mixtos e irregulares en el estado de Durango. Revista Mexicana de Ciencias Forestales, 11(59), 141-162. doi: $10.29298 /$ rmcf.v11i59.614
Füldner, K. (1995). Zur Strukturbeschreibung in Mischbeständen. Forstarchiv, 66, 149-161.

Gadow, K. von, Hui, G., \& Albert, M. (1998) Das Winkelmass - ein Strukturparameterzur Beschreibung der Individualverteilung in Waldbeständen. Centralblatt für das Gesamte Forstwesen, 115, 1-9.

Gadow, K. von, Real, P. \& Álvarez, J. G. (2001) Modelización del crecimiento y la evolución de los bosques. IUFRO World Series 12, 242.

Gadow, K. von, Sánchez, S., \& Álvarez J. G. (2007) Estructura y crecimiento del bosque. Universidad de Göttingen, Alemania.

Galván-Moreno, R. (2016). Manual para la identificación de Altos Valores de Conservación en bosques templados de México. La experiencia del suroeste del estado de Chibuahua. Programa de las Naciones Unidas para el Desarrollo y Comisión Nacional Forestal Editado e impreso en México. Primera edición, octubre de 2016. Recuperado de https://www.gob.mx/cms/uploads/attachment/file/185985/M anual_para_la_identificacion_de_Altos_Valores_de_Conservaci on_en_bosques_templados_de_Mexico.pdf

García García, S. A., Narváez Flores, R., Olivas García, J. M., \& Hernández Salas, J. (2019). Diversidad y estructura vertical del bosque de pino-encino en Guadalupe y Calvo, Chihuahua. Revista Mexicana de Ciencias Forestales, 10(53), 41-63. doi: 10.29298/rmcf.v10i53.173

García Y., Olivares, L., \& Ramos, J. (2013). Estructura y composición arbórea de un fragmento de bosque mesófilo de montaña en el estado de Veracruz. Revista Chapingo Serie Ciencias Forestales y del Ambiente 19, 91-101. doi: 10.5154/r.rchscfa.2012.03.025

Getzin, S., Wiegand, T., Wiegand, K., \& He, F. Heterogeneity influences spatial patterns and demographics in forest stands. Journal of Ecology, (2008), 96, 807-820. doi: 10.1111/j.13652745.2008.01377.x

Graciano-Ávila, G., Alanís-Rodríguez, E., Aguirre-Calderón, Ó. A., González-Tagle, M. A., Treviño-Garza, E. J., \& Mora-Olivo, A. (2019). Composición y estructura del estrato arbóreo bajo diferentes tratamientos silvicolas en un ecosistema forestal de la sierra madre occidental (México). Tesis Doctoral. Universidad Autónoma de Nuevo León. Nuevo León, México.

Graciano-Ávila, G., Alanís-Rodríguez, E., Rubio-Camacho, E. A., Valdecantos-Dema, A., Aguirre-Calderón, O. A., GonzálezTagle, M. A., ..., \& Mora-Olivo, A. (2020). Composición y estructura espacial de cinco asociaciones de bosques de Pinus durangensis. Madera y Bosques, 26(2). doi: $10.21829 /$ myb.2020.2621933 
Gu, H., Li, J., Qi, G., \& Wang, S. (2019). Species spatial distributions in a warm-temperate deciduous broad-leaved forest in China. Journal of Forestry Research, 31, 1-8.

Hermann, R. K. (1985). The Genus Pseudotsuga: Ancestral bistory and past distribution. Corvallis, OR: Oregon State University

Hernández, J., Aguirre, O. A., Alanís, E., Jiménez, J., Treviño, E. J., González, M. A., Lujan, C., Olivas, J. M., \& Domínguez, L. A. (2013). Efecto del manejo forestal en la diversidad y composición arbórea de un bosque templado del Noreste de México. Revista Chapingo Serie Ciencia Forestal y del Ambiente 19:189-200. doi: 10.5154/r.rchscfa.2012.08.052

Hui, G. Y., \& Hu, Y. B. (2001). Measuring species spatial segregation in mixed forest. Forest Research, 14, 23-27.

Kulik, N. C. P., de Molas, L. P., \& Rodríguez, S. M. A. (2019). Análisis estructural de un bosque de la Ecorregión Alto Paraná, Paraguay. Investigación Agraria, 20(2), 127-135.

Lamprecht, H. (1990). Silvicultura en los trópicos: Los ecosistemas forestales en los bosques tropicales y sus especies arbóreas, posibilidades y métodos para un aprovechamiento sostenido. Carrillo, A. (Trad.). Eschborn, Alemania: GTZ (Cooperación Técnica Alemana).

Lin, H. T., Lam, T. Y., Gadow, K. von, \& Kershaw Jr, J. A. (2020). Efectos de los diseños de parcelas anidadas en la evaluación de atributos de rodales, diversidad de especies y estructuras forestales espaciales. Forest Ecology and Management, 457, 117658. doi: 10.1016/j.foreco.2019.117658

Mair, P., Wilcox, R., \& Schoenbrodt, F. (2011). WRS2: a collection of robust statistical methods. Recuperado de https://cran.rproject.org/web/packages/WRS2/index.html

Mápula-Larreta, M., López-Upton, J., Vargas-Hernández, J. J., \& Hernández-Livera, A. (2007). Reproductive indicators in natural populations of Douglas-fir in Mexico. Biodiversity and Conservation, 16(3), 727-742.

Mápula-Larreta, M., López-Upton, J., Vargas-Hernández, J., \& Hernández-Livera, A. (2008). Germinación y vigor de semillas en Pseudotsuga menziesii de México. Ra Ximhai, 4(1), 119-134. doi: 10.35197/rx.04.01.2008.07.mm

Mena-Mosquera, V., Andrade C., H., \& Torres-Torres, J. (2019). Composición florística, estructura y diversidad del bosque pluvial tropical de la subcuenca del río Munguidó, Quibdó, Chocó, Colombia. Entramado, 16(1), 204-215. doi: 10.18041/19003803/entramado.1.6109

Marie-Agnès, M., \& Audrey, R. (2003). A model to assess relationships between forest dynamics and spatial structure. Journal of Vegetation Science, 14(6), 823-834. doi: 10.1111/j.1654-1103.2003.tb02215.x
Ogawa, H., Yoda, K., Ogino, K. \& Kira, T., (1965). Comparative ecological studies on three main types of forest vegetation in Thailand II Plant biomass. Nature and Life Southeast Asia, 4: 49-80

Pommerening, A., \& Stoyan, D. (2006). Edge-correction needs in estimating indices of spatial forest structure. Canadian Journal of Forest Research, 36,1723-1739. doi: 10.1139/x06-060

Pommerening, A., Wang, H., \& Zhao, Z. (2020). Global woodland structure from local interactions: new nearest-neighbour functions for understanding the ontogenesis of global forest structure. Forest Ecosystems, 7(1), 1-11. doi: 10.1186/s40663-02000224-5

Pretzsch, H. (2009). Forest dynamics, growth, and yield. In Forest dynamics, growth and yield. Springer, Berlin, Heidelberg.

R Core Team. (2019). R: A language and environment for statistical computing. R Foundation for Statistical Computing. Vienna, Austria: R Foundation for Statistical Computing. Recuperado de https://www.r-project.org/

Rubio-Camacho, E. A., González-Tagle, M. A., Himmelsbach, W., Ávila-Flores, D. Y., Alanís-Rodríguez, E., \& Jiménez-Pérez, J. (2017). Patrones de distribución espacial del arbolado en un bosque mixto de pino-encino del noreste de México. Revista Mexicana de Biodiversidad, 88(1), 113-121. doi: 10.1016/j.rmb.2017.01.015

Rzedowski, J. (1978). Vegetación de México: México. Editorial Limusa.

Schwendtner, O., Recalde, I., Alcalde, J. T., Gómez, J., \& Cárcamo, S. (2005). Importancia de los árboles senescentes y la madera muerta en la gestión de los bosques naturales. Presentado en el IV Congreso Forestal Español.

Solís Sánchez, J., Blanco García, A., Sáenz Romero, C., Méndez Toribio, M., \& López Toledo, L. (2018). El cambio climático y las coníferas mexicanas. CONABIO. Biodiversitas, 141:7-10.

Torres, D. (2006). Evaluación de la regeneración natural de tres poblaciones de Pseudotsuga sp. en el estado de Tlaxcala. Tesis de licenciatura, Universidad Autónoma de Tlaxcala. Tlaxcala, Tlaxcala. México.

Organización de las Naciones Unidas para la Educación, la Ciencia y la Cultura [Unesco] (1980). Ecosistemas de los bosques tropicales. Informe sobre el estado de conocimientos. París: Unesco. Recuperado de https://books.google.com.mx/books/about/Ecosistemas_de_1 os_bosques_tropicales.html?id=gabKnQEACAAJ\&redir_esc $=y$

Valverde, T., \& Silvertown, J. (1997). Canopy closure rate and forest structure. Ecology, 78(5), 1555-1562. doi: 10.2307/2266148

Ventura Ríos, A., López Upton, J., Vargas Hernández, J. J., \& Guerra de la Cruz, V. (2010). Caracterización de Pseudotsuga meñiesii (MIRB.) Franco en el centro de México: Implicaciones para su 
conservación. Revista Fitotecnia Mexicana, 33(2), 107-116. doi: 10.35196/rfm.2010.2.107

Velasco-García, M. V., López-Upton, J., Angeles-Pérez, G., VargasHernández, J., \& Guerra-de la Cruz, V. (2007). Dispersión de semillas de Pseudotsuga menziesii en poblaciones del centro de México. Agrociencia, 41(1), 121-131.

Wédjangnon, A. A., Kuiga, N. B. S., Houêtchégnon, T., \& Ouinsavi, C. A. (2020). Spatial distribution and interspecific association patterns between Mansonia altissima A. Chev., Ceiba pentandra (L.) Gaertn and Triplochiton scleroxylon K. Schum. in a moist semideciduous forest. Annals of Forest Science, 77(1), 1-11.

Wilcox, R. R. (2011). Introduction to robust estimation and hypothesis testing. Academic press.

Yang, X., Yan, H., Li, B., Han, Y., \& Song, B. (2018). Spatial distribution patterns of Symplocos congeners in a subtropical evergreen broad-leaf forest of southern China. Journal of Forestry Research, 29(3), 773-784.

Yu, H., Wiegand, T., Yang, X., \& Ci, L. (2009). The impact of fire density-dependent mortality on the spatial patterns of a pine forest in the Hulun Buir sandland, Inner Mongolia, China. Forest Ecology and Management 257, 2098-2017. doi: 10.1016/j.foreco.2009.02.019
Zhang, G., Hui, G., Hu, Y., Zhao, Z., Guan, X., Gadow, K. von, \& Zhang, G. (2019). Designing near-natural planting patterns for plantation forests in China. Forest Ecosystems, 6(1), 28. doi: 10.1186/s40663-019-0187-x

Manuscrito recibido el 21 de agosto de 2020

Aceptado el 22 de junio de 2021

Publicado el 23 de diciembre de 2021

Este documento se debe citar como:

García-García, S. A., Alanís-Rodríguez, E., Rubio-Camacho, E. A., Aguirre-Calderón, O. A., Treviño-Garza, E. J., \& GracianoÁvila, G. (2021). Patrones de distribución espacial del arbolado en un bosque de Pseudotsuga menziesii en Chihuahua, México. Madera y Bosques, 27(3), e2732242. doi: 10.21829/myb.2021.2732242

Madera y Bosques por Instituto de Ecología, A.C. se distribuye bajo una Licencia Creative Commons Atribución-NoComercialCompartirlgual 4.0 Internacional. 